\title{
Key transitions in animal evolution
}

\author{
Rob DeSalle ${ }^{1, *}$ and Bernd Schierwater,,$\dagger$
}

${ }^{\star}$ American Museum of Natural History, New York, Division of Invertebrate Zoology 79 St at Central Park West, New York, NY 10024; †ITZ, Ecology \& Evolution, TiHo Hannover, Bünteweg 17d, D-30559 Hannover, Germany

Synopsis In order to address this subject further and to assess progress in the examination of animal origins and transitions, an international group of scientists was convened at the Society for Comparative Biology meeting in January 2007.

\section{Introduction}

"That theory is worthless. It isn't even wrong!"

Wolfgang Pauli

The origin of multicellular animals is one of those difficult and delicate biological problems. Many ideas have been proffered and many hypotheses have been tested with respect to the subject. In order to address this subject further and to assess progress in the examination of animal origins and transitions, an international group of scientists was convened at the Society for Comparative Biology meetings in January of 2007. The meeting sought to bring together experts in many areas of research on the origin of multicellular animals from a variety of biological perspectives. In the SICB symposium "Key Transitions in Animal Evolution" the current views on old and new debates of important concepts were presented, discussed and evaluated. In order to put the papers delivered in this symposium into context some background information is presented in this introduction.

\section{Animals and Bauplans}

"All animals are equal, but some animals are more equal than others."

George Orwell

Multicellular animals (metazoans) almost certainly derive from a cum grano salis "single" celled protozoan ancestor. Metazoa-by definition-harbor more than one somatic cell type and can be classified into basal (or diploblastic) and derived (or triploblastic) groups. These groups differ by the existence of three (triploblasts) or two germ layers only (diploblasts). It is generally accepted that triploblasts derive from a diploblastic ancestor, implying that multicellular animals evolved from simple to more complex (anagenetic evolution). The basal (diploblastic) metazoans consist of sponges, placozoans, cnidarians, and ctenophores. The higher (derived triploblastic) metazoan groups are represented by two lines which differ by the fate of the blastoporus. In the Deuterostomia lineage the blastoporus becomes the anus of the adult, in the Protostomia the blastoporus becomes the mouth of the adult. Because of the definition of ventral and dorsal according to the position of the final mouth, Protostomia and Deuterostomia represent opposite dorsal-ventral Bauplans and endogenous versus exogenous skeleton systems.

The above transitions from single celled to multicellular, from diploblastic to triploblastic, and from protostomic to deuterostomic are-despite substantial efforts-not yet understood. To set the stage, the plenary paper presented by Pearse and Voigt (2007), discussed information on the involvement of Placozoa in key transitions in animal evolution. Quite interestingly, before we ask the question how Bauplan $\mathrm{x}$ got transformed into Bauplan y one may well ask "what is a Bauplan?" This important question was addressed by Extavour, et al. (2007) and Holland et al. (2007). In addition, the diploblast-triploblast grouping is discussed in detail in the papers by Boero et al. (2007) and Ball et al. (2007). In departures from discussions on transition from one body plan to another, Blackstone (2007), Plachetzki \& Oakley (2007) presented information on physiological transitions in the evolution of basal metazoans and Jacobs et al. (2007) presented information on the evolution of nervous systems.

\section{Comparative and evolutionary biology of early metazoans}

"It is better to know some of the questions than all of the answers."

James Thurber

From the symposium "Key Transitions in Animal Evolution" presented at the annual meeting of the Society for Integrative and Comparative Biology, January 3-7, 2007, at Phoenix, Arizona.

${ }^{1}$ E-mail: desalle@amnh.org

Integrative and Comparative Biology, volume 47, number 5, pp. 667-669

doi:10.1093/icb/icm042

Advanced Access publication June 18, 2007

(C) The Author 2007. Published by Oxford University Press on behalf of the Society for Integrative and Comparative Biology. All rights reserved.

For permissions please email: journals.permissions@oxfordjournals.org. 
Molecular systematics and EvoDevo research have provided the tools to examine in more depth the above key transitions in Bauplan in animal evolution. In principal, generation of phylogenetic trees and resolving the genetics of developmental pathways should together shed light on these key evolutionary events. At present, however, large data sets from both areas have provided conflicting scenarios and left open some very fundamental and crucial questions. The newer information has, however, crystallized key questions in animal transitions in the tree of life. What key innovations are relevant to the nature of the first animals? How have animal characteristics evolved since their origin? How do major changes in Bauplan relate to genetic changes? How can we best utilize such knowledge? These are a few of the questions that can be illuminated by comparative and evolutionary studies of animals; however, the most intensively studied systems represent relatively derived animals with derived characteristics. Yet basal diploblastic organisms have been widely under-represented and thus both the very origin of metazoan organization as well as the divergence of higher (triploblastic) animal organization are still subject of poorly supported speculations. Most recent evidence supports a basal position for Placozoa (Dellaporta et al. 2006), a possible sister-group relationship between Ctenophora and triploblasts (Bilateria), and derived dead-end positions for sponges and cnidarians, which include some of the best-studied basal metazoans (Collins et al. 2005). Outgroup definition and basal character states have a decisive influence on interpretations of character-state changes throughout the evolution of multicellular animals. The symposium papers addressing these issues include Pearse and Voigt (2007) Schierwater and DeSalle (2007) or in the context of choanoflagellates and sponges Lavrov (2007). Placing the question in a temporal context is important as shown by Collins and Cartwright (2007).

\section{Genomes and development}

"You could throw a whole genome at some problems and they will still probably be unresolved."

Max Telford

The rapidly increasing number of whole-genome projects has intensified the model system and research in molecular systematics. Genomic features require a comparative framework to be understood, and thus the choice of the right model organisms becomes particularly crucial (Martindale 2005).
Genes require a comparative classification and when animal genomes are sequenced, homologs need be assigned to various classes with some certainty (Copley et al. 2004). The interpretation of "gene homologs" has been a hot subject of debate and different researchers use crucial terms like "anterior/posterior and oral/aboral" in different, and interpretive ways (e.g., Abouheif 1999; Finnerty 2003), leading to conflicting views sometimes stemming from the same (or similar) data set(s) (Kamm et al. 2006; Chourout et al. 2006; Ryan et al. 2007). Great expectations derived from the study of Antp-class genes to unravel the evolution of axes formation, and thus the basic Bauplan for all major metazoan groups (e.g., Deutsch and Mouchel-Vielh 2003). While there is no doubt that regulatory developmental genes have played a major role in the key transitions in Bauplan evolution and will be crucial for unraveling evolutionary pathways of these transitions, these genetic data suffer from homology assessment problems. Yet these studies have fueled at least as many controversies as they have solved original questions. Not even the paradigm of homology of Hox genes between diploblasts and triploblasts has withstood experimental investigation as discussed by Schierwater and DeSalle (2007) in this issue. In this context the role of gene duplications has become a hot and controversial issue, as presented by Günter Wagner.

\section{Summary}

"You can observe a lot just by watchin'."

Yogi Berra

Understanding the changes that occurred at the base of the metazoan branch of the tree of life is all about "watchin"; but "watchin" what? During the proceedings of this symposium attendees were directed to "watch" a lot of things and we hope that the SICB symposium on Key Transitions in Animal Evolution and the papers generated from the symposium contribute to the observational richness on the metazoan branch of the tree of life. Through the papers delivered and the discussions that were generated as a response to the papers during the symposium, it is clear that many of the questions relevant to this part of the tree of life have not been answered and some have not even been addressed. However, the more we observe (watch) about this question, the richer our understanding becomes and more importantly the more well defined our questions and hypotheses become. 


\section{Acknowledgments}

We thank all speakers and particularly all authors for their intriguing contributions. The symposium was supported by a grant to BS and RD from the NSF (DEB-0706893) and by financial support from the American Microscopical Society, Division of Evolutionary Developmental Biology of the SICB, and the Society for Integrative and Comparative Biology. We also acknowledge Linda Walters and Vicki Pearse for their logistical support.

\section{References}

Abouheif E. 1999. Establishing homology criteria for regulatory gene networks: prospects and challenges. Novartis Found Symp 222:207-25.

Ball E, de Jong D, Schierwater B, Shinzato C, Hayward D, Miller D. Implications of cnidarian gene expression data for the origins of bilaterality: is the glass half full or half empty? Presented at the annual meeting of the Society for Integrative and Comparative Biology, 2007 Jan 3-7; Phoenix, AZ.

Blackstone N. 2007. A food's-eye view of the transition from basal metazoans to bilaterians. Integr Comp Biol 10.1093/ icb/icm056.

Boero F, Schierwater B, Piraino S. 2007. Cnidarian milestones in metazoan evolution. Integr Comp Biol. 10.1093/icb/icm041.

Chourrout D, Delsuc F, Chourrout P, Edvardsen SB, Rentzsch F, Renfer E, Jensen MF, Zhu B, de Jong P, Steele RE, Technau U. 2006. Minimal ProtoHox cluster inferred from bilaterian and cnidarian Hox complements. Nature 442:684-672.

Collins AG, Cartwright P, McFadden CS, Schierwater B. 2005. Phylogenetic context and basal metazoan model systems. Integr Comp Biol 45:585-94.

Collins AG, Cartwright P. Rocks and clocks: integrating fossils and molecules to date transitions in early animal evolution. Presented at the annual meeting of the Society for Integrative and Comparative Biology, 2007 Jan 3-7; Phoenix, AZ.

Copley RR, Aloy P, Russell RB, Telford MJ. 2004. Systematic searches for molecular synapomorphies in model metazoan genomes give some support for Ecdysozoa after accounting for the idiosyncrasies of Caenorhabditis elegans. Evol Dev 6:164-9.
Dellaporta SL, Xu A, Sagasser S, Jakob W, Moreno MA, Buss LW, Schierwater B. 2006. Mitochondrial genome of Trichoplax adhaerens supports Placozoa as the basal lower metazoan phylum. Proc Natl Acad Sci USA 103:8751-6.

Deutsch JS, Mouchel-Vielh E. 2003. Hox genes and the crustacean body plan. Bioessays 25:878-987.

Extavour C. 2007. Evolution of the bilaterian germ line: lineage origin and specification mechanism modulation. Integr Comp Biol. 10.1093/icb/icm027.

Finnerty JR. 2003. The origins of axial patterning in the metazoa: how old is bilateral symmetry? Int J Dev Biol 47:523-9.

Holland P, Okamura B, Jimenez-Guri E. Origin and evolution of a myxozoan worm. Presented at the annual meeting of the Society for Integrative and Comparative Biology, 2007 Jan 3-7; Phoenix, AZ.

Kamm K, Schierwater B, Jakob W, Miller D. 2006. Axial patterning and diversification in the Cnidaria predate the Hox system. Curr Biol 16:920-6.

Jacobs DK, Nichols S, Hartenstein V, Nakanishi N, Yuan D. Origins of sensory and neural organization in basal Metazoa. Presented at the annual meeting of the Society for Integrative and Comparative Biology, 2007 Jan 3-7; Phoenix, AZ.

Lavrov D. Key transitions in animal evolution: a mitochondrial DNA perspective. Presented at the annual meeting of the Society for Integrative and Comparative Biology, 2007 Jan 3-7; Phoenix, AZ.

Martindale MQ. 2005. The evolution of metazoan axial properties. Nat Rev Genet 6:917-27.

Pearse V, Voigt O. 2007. Field biology of placozoans (Trichoplax): distribution, diversity, biotic interactions. Integr Comp Biol. 10.1093/icb/icm015.

Plachetzki D, Oakley T. Key transitions during animal phototransduction evolution: Novelty, tree thinking, co-option and co-duplication. Integr Comp Biol. 0.1093/ icb/icm045.

Ryan JF, Mazza ME, Pang K, Matus DQ, Baxevanis AD, Martindale MQ, Finnerty JR. 2007. Pre-bilaterian origins of the Hox cluster and the Hox code: evidence from the sea anemone, Nematostella vectensis. PLoS ONE 2:1-23.

Schierwater B, DeSalle R. 2007. Can we ever identify the urmetazoan? Integr Comp Biol. 10.1093/icb/icm040. 